Article

\title{
Spiritual Well-Being as a Component of Health-Related Quality of Life: The Functional Assessment of Chronic Illness Therapy—Spiritual Well-Being Scale (FACIT-Sp)
}

\section{Jason M. Bredle ${ }^{1, *}$, John M. Salsman ${ }^{2}$, Scott M. Debb ${ }^{1}$, Benjamin J. Arnold ${ }^{1}$ and David Cella ${ }^{2}$}

1 FACIT / 381 S. Cottage Hill Avenue, Elmhurst, IL 60126, USA; E-Mails: sdebb@facit.org (S.M.D.); barnold@facit.org (B.J.A.)

2 Robert H. Lurie Comprehensive Cancer Center of Northwestern University; Department of Medical Social Sciences, Feinberg School of Medicine, Northwestern University, 710 N. Lake Shore Drive, Suite 729, Chicago, IL 60611, USA; E-Mails: j-salsman@northwestern.edu (J.M.S.); d-cella@northwestern.edu (D.C.)

* Author to whom correspondence should be addressed; E-Mail: jbredle@facit.org; Tel.: +1-773-807-9094; Fax: +1-509-461-7017.

Received: 22 November 2010; in revised form: 25 February 2011 / Accepted: 9 March 2011 / Published: 15 March 2011

\begin{abstract}
The Functional Assessment of Chronic Illness Therapy-Spiritual Well-Being (FACIT-Sp-12) is a 12-item questionnaire that measures spiritual well-being in people with cancer and other chronic illnesses. Cancer patients, psychotherapists, and religious/spiritual experts provided input on the development of the items. It was validated with a large, ethnically diverse sample. It has been successfully used to assess spiritual well-being across a wide range of religious traditions, including those who identify themselves as "spiritual yet not religious." Part of the larger FACIT measurement system that assesses multidimensional health related quality of life (HRQOL), the FACIT-Sp-12 has been translated and linguistically validated in 15 languages and has been used in dozens of studies examining the relationships among spiritual well-being, health, and adjustment to illness.
\end{abstract}

Keywords: spiritual well-being; health related quality of life (HRQOL); FACIT-Sp 


\section{Abbreviations}

HRQOL: health-related quality of life; PRO: patient-reported outcomes; FACIT-Sp: Functional Assessment of Chronic Illness Therapy - Spiritual well-being; FACT-G: Functional Assessment of Cancer Therapy - General.

\section{Introduction}

In recent decades, spiritual well-being has become of central importance in many health care settings as researchers continue to study its effects upon health [1]. However, no clear consensus exists between the definition of religion and the concept of spirituality in the current literature. For example, some researchers posit that religion is concerned primarily with learning from the experience of others and spirituality is concerned with urging one to initiate his or her own experiences [2]. Others suggest that religion can be thought of as a set of shared beliefs and practices and spirituality the application of those beliefs and practices [3]. Part of the problem is due to the polarization of the terms. Zinnbauer et al. [4] have noted the following comparisons: religion is considered external and institutional, whereas spirituality is considered personal and relational; religion is viewed as a static, substantive entity, whereas spirituality is dynamic and functional; and religion is depicted negatively, whereas spirituality is depicted positively. They argue that dichotomizing these constructs in this fashion obscures the full character of each as religion and spirituality both include helpful and harmful, traditional and progressive, personal and institutional elements [4]. Similarly, Hill et al. [1] suggest that religion and spirituality are multidimensional phenomena that overlap with one another but also retain distinctive elements (see Fetzer Institute, 1999 [5]; Koenig et al., 2001 [6] for a detailed presentation of the multidimensional aspects of religion and spirituality). In health care settings, it is important to understand the significance of this overlap and its relation to both physical and mental health outcomes in order to improve patient care. In fact, some models of health include spiritual wellbeing as one component of health-related quality of life (HRQOL) [7,8]. This biopsychosocial-spiritual model of health views spiritual well-being as an essential component of HRQOL, related to but distinct from more traditional HRQOL domains: physical, mental, and social well-being. This model may be particularly useful when applied to healthcare settings or for patients suffering from serious illnesses, as being able to accurately and reliably measure patients' spiritual well-being, or the degree to which patients' spirituality can help them make sense of their lives, and feel whole, hopeful and peaceful even in the midst of a serious illness, can help both clinicians and researchers with regards to clinical conceptualization and subsequent treatment planning.

In the present study, the aim was to gather background information on the development of the FACIT-Sp, as well as subsequent empirical data from studies that have employed the measure as primary or secondary predictors or as outcome variables. The scope was not intended to be an exhaustive review of every study that uses the FACIT-Sp in any capacity. Rather, the inclusion criteria were studies that employed the FACIT-Sp as an integral component where results enhanced our understanding of the FACIT-Sp, of spiritual well-being within chronic illness populations, or both. To this end, studies with relatively small samples that significantly lacked generalizability were excluded, as were studies that included the FACIT-Sp in a supplementary fashion. Also, reporting of statistical 
significance has been limited to data that has directly impacted implications for the FACIT-Sp. Finally, while several measures of spiritual well-being exist, the focus of this review is on spiritual well-being as assessed by the FACIT-Sp.

Articles were identified as part of a comprehensive literature review that evaluated peer-reviewed publications from the medical and social science fields. Commonly used electronic databases such as Medline, PsychInfo, and Academic Search Complete facilitated the process of compiling articles for review, and specific keywords helped winnow the articles that directly and indirectly related to spiritual well-being. Search terms included (health-related) quality of life, patient reported outcomes, questionnaire, survey, well-being, health status, spirituality, religion, FACT (Functional Assessment of Cancer Therapy), and FACIT (Functional Assessment of Chronic Illness Therapy). Additionally, references from seminal articles on spiritual well-being and articles referencing the FACIT-Sp directly were scrutinized for possible linkages to other substantive publications. Following is a summary of the peer-reviewed literature that highlights the statistical significance and clinical application of the FACIT-Sp to date.

\section{Spiritual Well-Being and the FACIT-Sp}

The FACIT-Sp was developed in the 1990s to address the need for a brief, broad measure of spiritual well-being with content not limited to any one religious or spiritual tradition. In addition to the four subscales of the FACT-G (physical well-being, social/family well-being, emotional well-being and functional well-being), it consists of 12 items and three sub-domains of spiritual well-being, which help facilitate an in-depth exploration of the components that constitute spiritual well-being (peace, meaning, and faith). The spiritual well-being subscale alone is referred to as the FACIT-Sp-12 (FACIT-Sp-12; see Table 1). It has also been adapted for use with the general population with a nonillness version, and an expanded version also exists, which includes an additional 11 items added to the original 12 .

Table 1. FACIT-Sp-12 (version 4).

\begin{tabular}{|c|c|c|c|c|c|c|}
\hline & & $\begin{array}{l}\text { Not at } \\
\text { all }\end{array}$ & $\begin{array}{l}\text { A little } \\
\text { bit }\end{array}$ & $\begin{array}{l}\text { Some } \\
\text {-what }\end{array}$ & $\begin{array}{l}\text { Quite } \\
\text { a bit }\end{array}$ & $\begin{array}{l}\text { Very } \\
\text { much }\end{array}$ \\
\hline Sp1 & 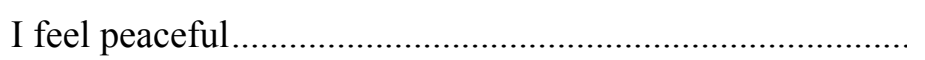 & & 1 & 2 & 3 & 4 \\
\hline Sp2 & I have a reason for living & & 1 & 2 & 3 & 4 \\
\hline Sp3 & 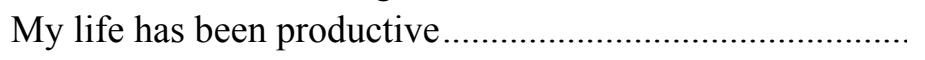 & & 1 & 2 & 3 & 4 \\
\hline Sp4 & 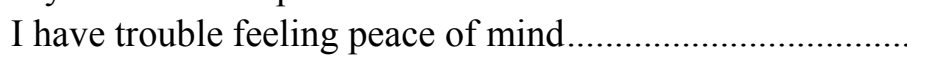 & & 1 & 2 & 3 & 4 \\
\hline Sp5 & 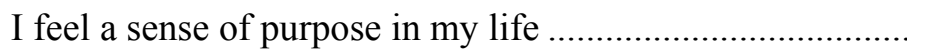 & 0 & 1 & 2 & 3 & 4 \\
\hline Sp6 & I am able to reach down deep into myself for comfort ......... & & 1 & 2 & 3 & 4 \\
\hline Sp7 & 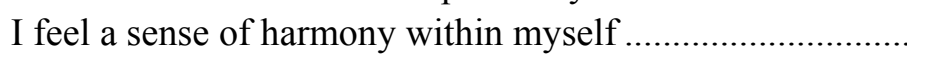 & & 1 & 2 & 3 & 4 \\
\hline Sp8 & 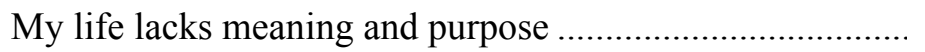 & 0 & 1 & 2 & 3 & 4 \\
\hline Sp9 & 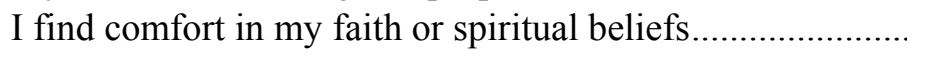 & & 1 & 2 & 3 & 4 \\
\hline Sp10 & 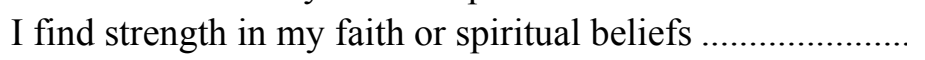 & & 1 & 2 & 3 & 4 \\
\hline Sp11 & My illness has strengthened my faith or spiritual beliefs..... & & 1 & 2 & 3 & 4 \\
\hline Sp12 & $\begin{array}{l}\text { I know that whatever happens with my illness, things } \\
\text { will be okay }\end{array}$ & & 1 & 2 & 3 & 4 \\
\hline
\end{tabular}

Above is a list of statements that other people with your illness have said are important. Please circle or mark one number per line to indicate your response as it applies to the past 7 days. 
Table 2. FACIT-Sp-12 Scoring Guidelines (Version 4).

\begin{tabular}{|c|c|c|c|c|c|}
\hline Subscale & Item Code & Reverse item & & Item response & Item Scor \\
\hline \multirow[t]{2}{*}{ Meaning } & $\mathrm{Sp} 2$ & 0 & + & & $=$ \\
\hline & $\mathrm{Sp} 3$ & 0 & + & & $=$ \\
\hline \multirow[t]{2}{*}{ Score range: $0-16$} & Sp5 & 0 & + & & $=$ \\
\hline & Sp8 & 4 & - & & $=$ \\
\hline
\end{tabular}

Sum individual item scores:

Multiply by 4:

Divide by number of items answered: =Meaning subscale score

$\begin{array}{llllll}\text { Peace } & \text { Sp1 } & 0 & + & - & = \\ & \text { Sp4 } & 4 & - & - & = \\ \text { Score range: } 0-16 & \text { Sp6 } & 0 & + & - & =\end{array}$

Sum individual item scores:

Multiply by 4:

Divide by number of items answered:

$=$ Peace subscale score

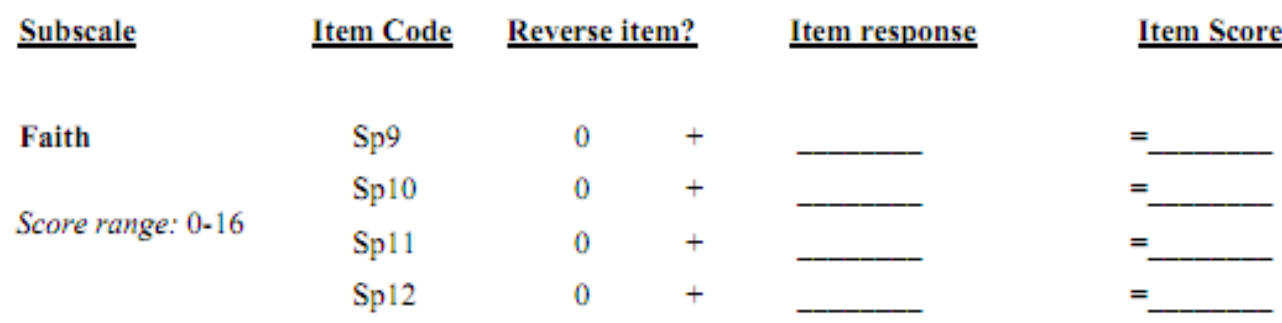

Sum individual item scores:

Multiply by 4:

Divide by number of items answered: =Faith subscale score

To Derive a FACIT-Sp12 total score:

Score range: 0.48

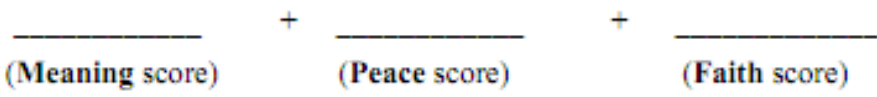

$=$

=FACIT-Sp12 Total score

Instructions: 1. Record answers in "item response" column. If missing, mark with an X; 2. Perform reversals as indicated, and sum individual items to obtain a score; 3 . Multiply the sum of the item scores by the number of items in the subscale, then divide by the number of items answered. This produces the subscale score; 4 .

The higher the score, the better the QOL/spiritual well-being.

All of the FACIT-Sp questionnaires were designed for self-administration and use a 5-point Likerttype scale to measure patient-reported HRQOL $(0=$ Not at all; $1=$ A little bit; 2 = Somewhat; $3=$ Quite a bit; and $4=$ Very much). The recall period for each question is seven days. Questions were written at the fourth grade-reading level as measured by the Lexile Framework [9]. Formatting was done to enable self-administration with minimal burden. The option to complete the questionnaire by 
interview decreases burden for patients whose condition or mood preclude them from completing the questionnaire on their own (e.g. due to fatigue, depression, or poor eyesight), although this may increase completion time. Supervision (review of the items) is recommended when the patient has completed the questionnaire to ensure that all questions have been answered to the best of the respondent's capability. The questionnaire is easy to score using the scoring template (Table 2), and scoring algorithms are also available for use with commonly used data analysis programs. To date, the English version of the FACIT-Sp has also been translated and linguistically validated in Arabic, Chinese (Simplified and Traditional), Danish, Dutch, Farsi, French, German, Italian, Japanese, Korean, Norwegian, Portuguese, Spanish, and Swedish. Please see www.facit.org for an up to date list on language availability for all FACIT scales and scoring protocols.

\section{Validation and Application of the FACIT-Sp}

\subsection{Instrument Validation and Factor Structure}

The FACIT-Sp was initially developed and validated using two independent samples of cancer patients and survivors [10]. A sample of predominantly cancer patients were recruited from the United States and Puerto Rico $(\mathrm{N}=1,617)$ and administered multiple general measures of HRQOL as well as the FACIT-Sp. The internal reliability of the subscales was good $(\alpha=0.81-0.88)$ and there were moderate to strong correlations between the total FACIT-Sp subscale score and the other HRQOL instruments, including the FACT-G scale $(r=0.58)$ and different domains of the Profile of Mood States (POMS; $r=-0.36-0.54$ for negatively-oriented domains such as Depression and Confusion, and $r=0.42$ for the Vigor domain). A second study was then performed to evaluate the FACIT-Sp's relation to existing measures of religion and spiritual well-being. Data were analyzed from 131 participants in the context of a larger study investigating fatigue and HRQOL for patients beginning chemotherapy. Internal reliability was comparable to study 1 and the FACIT-Sp correlated moderately to strongly with six other measures of spiritual well-being and religion, ranging from the Cancer Patient Behavior Survey (CPBS) Satisfaction With Religion $(r=0.25)$ to the Spiritual Beliefs Inventory (SBI) $(r=0.48)$. It was concluded that the FACIT-Sp is a brief, reliable and valid measurement of spiritual well-being that may be particularly useful in assessing the role of both religious and non-religious spiritual well-being in HRQOL [7,11].

Following the development and validation of the FACIT-Sp, an extended version - the FACIT-SpEx-was designed to include an additional 11 items concerning forgiveness, connectedness, and appreciation. In a study evaluating the additional items' psychometric properties, Cotton et al. [12] administered questionnaires assessing religiosity and spiritual well-being, HRQOL, depression, and other psychosocial variables to 450 HIV/AIDS patients. An exploratory factor analysis was performed and internal consistency/convergent validity assessed for each of the identified factors and the overall scale. Three factors - meaning/peace, faith, and relational-had Eigenvalues $>1.0$, suggesting a three factor structure. Internal consistency was high for the overall 23-item scale $(\alpha=0.95)$ and for its three subscales (meaning/peace, $\alpha=0.90$; faith, $\alpha=0.93$; relational, $\alpha=0.83$ ). Relational correlated as expected with measures of HIV-related HRQOL, social support, and self-esteem, ( $r$ ranging from 0.41 to $0.49 ; \mathrm{p}<0.01)$. The authors concluded that the FACIT-Sp-Ex appears to have a third subscale that 
assesses relational aspects of spiritual well-being and that future examination of the psychometric properties of the full 23-items is necessary in order to generalize these findings to other patient samples and provide further evidence of the unique contributions of the Relational subscale of the FACIT-Sp-Ex to spiritual well-being.

The importance of spiritual well-being as a component of HRQOL and its clinical utility has been illustrated by a study of a diverse sample of 1,610 cancer and HIV patients [13]. The FACIT-Sp was used to measure spiritual well-being in this study, and results indicated that spiritual well-being was associated with overall HRQOL $(r=0.48)$ to the same degree as various sub-domains of HRQOL, physical well-being $(r=0.47)$ in particular. The clinical utility of measuring spiritual well-being was also observed in that spiritual well-being was found to be related to enjoyment of life, despite the presence of significant symptoms. Overall, results suggested a biopsychosocial-spiritual model for assessment of HRQOL might be more comprehensive than not including the spiritual well-being dimension.

The original factor analyses of the FACIT-Sp supported two factors: meaning/peace and faith. Because meaning suggests a cognitive aspect of spiritual well-being and peace an affective component, in a subsequent study, Canada et al. hypothesized a three-factor solution: meaning, peace, and faith [14]. Two hundred and forty females previously diagnosed with cancer and at least five years postdiagnosis completed a telephone interview consisting of the FACIT-Sp and several other questionnaires. During analysis, items were assigned to the two-factor model as previously established in the literature. An alternative three-factor model was then proposed. The analysis of this model provided more information about each item and about the overall model: it was found that the peace factor was only correlated with mental health scores, meaning was associated with both physical and mental health scores, and faith was negatively associated with mental health scores. Specifically, using partial correlations, the Peace factor was moderately related to mental health $(r=0.53, \mathrm{p}<0.001)$; Meaning was related only modestly to physical $(r=0.18, \mathrm{p}<0.01)$ and mental $(r=0.17, \mathrm{p}<0.01)$ health; and Faith was negatively but modestly associated with mental health $(r=-0.17, \mathrm{p}<0.05)$. In this study and a follow-up study examining the factor structure of the FACIT-Sp [15], the three-factor model proved to be a psychometric improvement over the two-factor model, enabling a more detailed examination of the differing dimensions of religion/spiritual well-being to HRQOL.

A more recent study was conducted intending to replicate previous research suggesting that the three-factor model of meaning/peace/faith is psychometrically superior to a two-factor model [16]. Building on the assumption of a valid three-factor model, the study aimed to assess whether the meaning and peace subscales of the FACIT-Sp functioned differently vis-à-vis physical and mental health outcomes. In performing the analysis, archival data from four samples totaling 2,810 patients with HIV/AIDS, cancer, and those who underwent bone marrow transplantation were gathered. Participants were asked to complete the FACIT-Sp along with several other physical and mental health measures. Results of the confirmatory factor analysis indicated that the three-factor models had a better absolute fit than the two-factor models in all four samples (Comparative Fit Index and Tucker-Lewis Index consistently higher for the three factor solutions to each sample). Across all of the samples, meaning and peace were highly correlated with one another $(r=0.60-0.88)$, and were correlated in the same direction with multiple health outcomes. To assess the degree to which Meaning or Peace carries incremental validity of the other, the authors conducted a pair of hierarchical regressions in which they 
first entered Peace followed by Meaning (to assess the unique impact of Meaning) and then reversed the order of entry such that they entered Meaning followed by Peace (to assess the unique impact of Peace). With a few exceptions, peace typically accounted for a greater portion of unique variance than Meaning. The authors concluded that a three-factor model consisting of faith, meaning, and peace domains provided a psychometrically better fit for the FACIT-Sp than a two-factor model where the meaning and peace constructs were combined as a single factor. The 3-factor model may also provide greater clarity in interpreting relationships between spiritual well-being and important indicators of physical and mental well-being. This was also consistent with previous conclusions reached by other researchers [15].

\subsection{Spiritual Well-Being as a Perspective on Psychosocial Adaptation}

The development and validation of the FACIT-Sp has led to its use in a number of different research studies exploring the links between spiritual well-being and psychosocial adaptation. These studies have helped expand our understanding of the relationship between HRQOL, spiritual wellbeing and psychological distress. This includes an increased understanding of the relationship between spiritual well-being and coping among patients with acute and chronic illness.

HRQOL in colorectal cancer survivors was examined in a study looking at the relationship between spiritual well-being and HRQOL outcomes, as well as whether the relationship between distress, spiritual well-being and HRQOL was best explained by a stress-buffering (interaction effect) or direct (main effects) model [17]. The FACIT-Sp and the Functional Assessment of Cancer TherapyColorectal cancer (FACT-C) were used to explore these objectives. Additional measures were used to assess general distress and cancer-specific distress. Regression analysis was conducted on 258 colorectal cancer survivors, and spiritual well-being (faith and meaning/peace) was found to have a positive association with HRQOL ( $r=0.249$ and 0.515 respectively), with meaning/peace a more robust indicator of HRQOL than faith. These findings were replicated in a second sample of 568 colorectal cancer survivors. Similar correlations were observed $(r=0.303$ for faith and 0.677 for meaning/peace). Across both samples, regression results generally supported a direct effects model of meaning/peace within the context of HRQOL assessment (i.e., in only one of the six regression models was a significant interaction obtained).

In another study aimed at clarifying the relationship between spiritual well-being, psychological adjustment, and HRQOL, a sample of 142 breast cancer patients was recruited to help clinicians examine the ways in which spirit, mind, and body could be connected to issues related to cancer care [8]. Using the FACIT-Sp and The Principles of Living Survey (PLS), results yielded a positive correlation between spiritual well-being and HRQOL $(r=0.48)$, even when controlling for psychological adjustment. Spiritual well-being was found to correlate negatively with some adjustment styles (i.e., helpless/hopeless; $r=-0.55$, anxious preoccupation; $r=-0.49$ and avoidance; $r=-0.21$ ) and positively with others (i.e., fatalism; $r=0.62$, fighting spirit; $r=0.46$ ), highlighting the importance of considering these components in HRQOL assessment.

The FACIT-Sp has also been used for cross-cultural research to better understand the determinants of self-reported HRQOL in Spanish-speaking cancer patients in South America [18]. In one study, a cross-sectional design was used with a sample of 309 patients in Uruguay. Psychological well-being 
was measured using the POMS-SF, and HRQOL and spirituality-related outcomes were evaluated using the FACIT-Sp, where the twelve item FACIT-Sp subscale score was used as a possible explanatory variable of patients' assessments of HRQOL. Five regression models were studied for the FACT-G total score and its four subscales. Five variables explained $32 \%$ of the variance of the FACT$\mathrm{G}$ total score: tumor stage, spiritual well-being, income, mood disorders, and mode of questionnaire administration. Results suggested that spiritual well-being was an important determinant of participants' assessments of overall HRQOL. Interestingly, of the patients surveyed, about $42 \%$ reported no current religious affiliation, which is typical to Uruguay but unlike the majority of South American nations. The authors found it noteworthy that spiritual well-being remained relevant despite this fact. They concluded that spiritual characteristics may be considered permanently embedded in a person's coping mechanisms, and inferred that this could lead to better HRQOL.

Adult survivors of hematopoietic stem-cell transplantation for malignant disease were also studied with regards to their HRQOL [19]. Six hundred and sixty-two participants completed a battery of questionnaires, including the FACIT-Sp and a healthy comparison group of 158 participants completed a parallel set of questionnaires. MANOVA results showed that the survivor group reported poorer status relative to the healthy group for all HRQOL outcomes; however, the survivor group reported more psychological and interpersonal growth. Mean effect size for the 24 outcome indices examined was considered clinically meaningful (0.36 standard deviation). The group effect for FACIT-Sp scores demonstrated a non-significant trend $(p=0.054)$, with the survivor group reporting poorer spiritual well-being.

Another examined psychosocial health to determine whether lower spiritual well-being in men with early stage prostate cancer was associated with lower HRQOL [20]. Two hundred and twenty-two participants were drawn from a program providing free prostate cancer treatment to indigent men, and validated measures including the FACIT-Sp were used to gather data on spiritual well-being, HRQOL, anxiety, symptom distress and emotional well-being. Univariate analyses indicated a consistent relationship between spiritual well-being and outcomes. Low spiritual well-being was associated with significantly worse physical and mental health, sexual function and urinary dysfunction than in patients with high spiritual well-being. The psychosocial variables (emotional well-being, symptom distress and anxiety) also reflected poorer adjustment in men with lower reported spiritual well-being. Multivariate analyses revealed that the peace and meaning subscale was responsible for better HRQOL scores as opposed to the faith component.

The associations among spiritual well-being and religiosity and depressive symptom severity was explored with a sample of 84 cancer patients and 78 AIDS/HIV patients with life expectancy of less than six months [21]. Multiple measures were used to assess cognitive impairment, depression, pain, performance status, physical well-being, and social support, and the FACIT-Sp was used to gauge spiritual well-being. Additional questions were asked to patients in order to assess religiosity and how spiritual they considered themselves to be. The study found a negative relationship between depression and spiritual well-being $(r=-0.40)$, where more spiritual individuals exhibited less depressive symptomology. This was particularly evident for depression items and the meaning/peace component of the FACIT-Sp $(r=-0.51)$, although religiosity only showed a small positive relationship with depression $(r=0.04)$. 
Another study geared towards the HIV/AIDS population looked at the direct and indirect effects of spiritual well-being/religion by looking at patient perceptions of living with HIV/AIDS [22]. Four hundred and fifty patients at various stages of HIV/AIDS illness were recruited, and spiritual wellbeing was measured using the FACIT-SP-Ex, as well as other survey instruments. Information related to health status, lifestyle, social support, self-esteem and healthy beliefs (optimism) was collected, and clinical data was considered. Patients who had attended college, were currently employed and identified themselves as having a religion were significantly more likely to say their lives had improved and these patients reported fewer distressing and depressive symptoms, and higher levels of optimism, self-esteem, social support and spiritual well-being.

Another study that examined how medical professionals perceive a patient's emotional distress and coping capacity included 90 pairs of Swedish cancer patients and their nurses [23]. Behavior, anxiety, and mood inventories were administered in conjunction with the Swedish version of the FACIT-Sp. When comparing results from both samples, the data suggested that nurses tended to overestimate patients' emotional needs by underestimating their resources to cope as well as their HRQOL, and nurse ratings were significantly higher in the areas of anxiety and depression ratings and lower in most areas of HRQOL including spiritual well-being (between group significance found at the 0.01 level for all domains except for the depression subscale of the Hospital Anxiety \& Depression Scale and the Physical Well-being subscale for the FACT-G). The clinical implications of this study build on previous findings suggesting that nurses in this population have difficulty conceptualizing their patient's anxiety and depression [24], as well as trouble making accurate assessments of patients' spiritual needs $[25,26]$.

Sample diversity in the form of mixed diagnoses was also incorporated into the methodology of one study that examined adjustment to the idea of coping with a long-term chronic illness. The FACIT-Sp was administered to a large sample of cancer patients $(\mathrm{N}=449)$ in order to investigate the relationship between spiritual well-being and HRQOL in an Australian population [26]. After examining bivariate correlations, spiritual well-being was found to have positive associations with HRQOL domains ( $r=0.19-0.62$, all ps $<0.001)$, with the meaning/peace component being more highly related to HRQOL than the faith component. Spiritual well-being was found to be uniquely predictive of HRQOL (i.e., "I am content with the quality of my life right now") beyond the standard HRQOL core domains of physical, social/family, and emotional well-being. Contrary to previous research, patients with high levels of spiritual well-being were not found to enjoy life to a greater degree in the presence of chronic illness symptoms than those with low levels of spiritual well-being. In terms of adjustment style, spiritual well-being was most highly correlated with fighting spirit and there was a negative association between spiritual well-being and helplessness/hopelessness. Fatalistic adjustment was negatively correlated with meaning/peace but positively correlated with faith. Taken as a whole, these results lend further support to using a biopsychosocial-spiritual model for HRQOL assessment.

In another study that employed an ethnically diverse sample, metastatic prostate cancer patients were recruited in order to explore the relationship between HRQOL and spiritual well-being $(\mathrm{N}=86)$ [27]. The FACIT-Sp was used to measure spiritual well-being and multiple instruments were used to measure general and disease specific HRQOL, including assessing patient satisfaction with their physician. Latino and African American men with less than a high school education were found to have the highest spiritual well-being scores. Spiritual well-being was significantly associated with both 
general and disease-specific markers of HRQOL. Higher spiritual well-being predicted higher scores in several HRQOL and psychosocial domains (at the $\mathrm{p}<0.05$ level). Significant interactions between meaning/peace and faith in physical and pain domains were also observed. Higher faith scores, in the absence of high meaning/peace scores, were negatively related to HRQOL, with meaning/peace scores correlating closely with HRQOL. Higher levels of spiritual well-being were related to better HRQOL and psychosocial function. It should be noted that the small sample size limited generalizability of the results, but it was still able to highlight the need for clinicians to be aware of the importance of spiritual well-being when working with patients coping with chronic illness.

Related to the idea of how a positive coping strategy can be linked to management of day-to-day symptomology is understanding the patient's life satisfaction. In one study looking at this, examination of the differences and factors related to HRQOL, life satisfaction, and spiritual well-being across rehabilitation and cancer patients was conducted with a sample of 136 rehabilitation (spinal cord injury, amputee, and polio) and 72 cancer (breast and prostate) patients [28]. HRQOL was measured using multiple general health status questionnaires, and spiritual well-being was measured using the FACIT-Sp. Cancer patients reported higher levels of spiritual well-being than rehabilitation patients. Age, marital status and work status were also found to be important factors associated with spiritual well-being. HRQOL and life satisfaction were also significantly related to spiritual well-being, with life satisfaction being more related to spiritual well-being and marital status than various domains of HRQOL alone. Spiritual well-being was not found to predict HRQOL or life satisfaction in cancer patients, but was found to be a predictor of both in rehabilitation patients. It should be noted, however, that these results were from bivariate analyses, which did not control for numerous demographic differences between subgroups.

While a substantial amount of literature has linked greater spiritual well-being to better health outcomes, negative spiritual well-being has been linked to poorer health outcomes as well. In several illness categories (e.g., cancer), negative religious coping (e.g., belief that one's illness is God's punishment or abandonment) was linked to increased risk for physical health decline. In one study, Edmonson et al. examined the role of religion in providing a sense of meaning [29]. Relationships among religious variables were studied in a sample of 237 cancer survivors who were administered the FACIT-Sp to determine whether religion's association with HRQOL is mediated by the degree to which it provides a sense of personal meaning on an individual. The faith-related items of the FACIT$\mathrm{Sp}$ were used to measure religiosity and the meaning/peace items of the FACIT-Sp were used to measure spiritual well-being. Hierarchical linear regression revealed that religiosity and spiritual wellbeing were conceptually and statistically distinct from one another in this sample, and they were differentially associated with HRQOL. It was posited that religion and its use as a means of coping or finding comfort is beneficial to the degree to which it facilitates meaning, coherence and purpose in patients with chronic illnesses.

Other research has examined the role of religious coping and spiritual struggle in relation to physiological, psychosocial, and components of HRQOL including spiritual well-being [30]. With a sample of 429 HIV/AIDS patients, chart review and other health inventories were used by Trevino et al. to assess physiological health status, psychiatric symptomology, social support, and religious coping strategies. The FACIT-Sp-Ex was used to measure spiritual outcomes. Correlations between positive religious coping or spiritual struggle and outcomes at baseline indicated that positive religious 
coping was associated with greater levels of spiritual well-being and self-esteem, whereas lower positive religious coping scores were associated with lower levels of spiritual well-being and selfesteem. Positive religious coping was a significant predictor of FACIT-Sp-Ex scores at follow up after controlling for FACIT-Sp-Ex scores at baseline; however, those with lower positive religious coping scores had increases in FACIT-Sp-Ex scores, as well. The authors posited two theories to explain the increase in positive religious coping. First, poorer health status and well-being may lead to spiritual struggle by challenging an individual's world view and creating confusion about spirituality, though empirical evidence of this is limited. A second possible interpretation is that spiritual struggle leads to lower levels of psychological, physiological, social, and spiritual well-being and positive religious coping leads to greater well-being, which was supported by the longitudinal results of the study. Positive religious coping was significantly associated with positive outcomes such as greater selfesteem $(\mathrm{p}<0.05)$, life satisfaction $(\mathrm{p}<0.01)$ and spiritual well-being $(\mathrm{p}<0.01)$.

One study of female breast cancer survivors examined long term markers of HRQOL, including spiritual well-being [31]. One hundred and twenty-seven women over the age of 70 who were cancer survivors for at least one year were administered the FACIT-Sp as part of a larger battery of questionnaires for the purpose of identifying the importance of psychosocial resources for older individuals coping with the stressors associated with breast cancer recovery. Spiritual well-being was a significant psychosocial factor associated with greater life satisfaction and lower symptomology related to negative effect in particular. The researchers noted that social support had a weaker than expected association with outcome variables. Overall, spiritual well-being was moderately correlated with life satisfaction $(r=0.318)$, depression $(r=-0.336)$, and general health perception $(r=0.305)$. It was concluded that the internalized nature of spiritual well-being may play a greater role with regards to patients experiencing higher levels of well-being long term, without necessarily suggesting that more immediate external factors were of less importance for survivors.

Finally, in two longitudinal studies, Yanez et al. [32] examined two components of spiritual wellbeing (meaning/peace and faith) and their interaction as predictors of psychological adjustment. Based on previous literature, the authors hypothesized that sense of meaning and peace would be more strongly linked than faith to improvements in adjustment following cancer. In the first study, 418 breast cancer patients were administered the FACIT-Sp as well as other measurements at baseline, six months and 12 months. Higher baseline meaning/peace and an increase in meaning/peace over 6 months predicted a decline in depressive symptoms and an increase in vitality across 12 months (all ps $<0.0001)$. Baseline faith predicted an increase in perceived cancer-related growth $(\mathrm{ps}<0.0001)$. The primary goal of the second study was to assess the generalizability of the first study's findings with a sample of male and female survivors of various cancers who were further from their diagnosis and primary treatment. One hundred and sixty-five participants were administered the FACIT-Sp and other indices of psychological adjustment. FACIT-Sp scores were similar to the first study, with an increase in meaning/peace related to improved mental health $(p<0.001)$ and lower cancer-related distress $(p<0.01)$. An increase in faith was related to increased cancer-related growth $(p<0.001)$. Both studies showed significant interactions between meaning/peace and faith in predicting adjustment. Findings suggest that when predicting adjustment, the ability to find meaning and peace functions as a positive resource for cancer survivors but faith may serve to facilitate or even hinder positive adjustment. 


\subsection{Spiritual Well-Being at the End-of-Life}

Clinicians working with chronic illness populations must be cognizant of their patients' end-of-life concerns, as these considerations can impact important healthcare decisions. Building on research suggesting that patients with little social support and low spiritual well-being tend to report a greater desire for a hastened death, another study examined HRQOL related to a desire for death among 326 patients with metastatic gastrointestinal or lung cancer [33]. These patients were administered attitude, hopelessness and depression inventories in conjunction with the FACIT-Sp. While a minority of palliative care patients report a high desire for hastened death, the researchers noted that patients reporting a heightened level of desire for death also had high levels of depression, hopelessness, physical symptomology, symptom frequency, and pain intensity, but also that they reported lower spiritual well-being. This was consistent with previous literature, and it was concluded that the results suggested that as a person progresses through the stages of a chronic disease, his or her desire for death often elevates, and that for many people, there could be an associated decline in spiritual well-being. To this end, multiple variables were significantly predictive of desire for hastened death, including functional status $(\mathrm{p}=0.046)$, depression $(\mathrm{p}<0.001)$, and hopelessness $(\mathrm{p}<0.0005)$.

Palliative care patients have been recruited and administered the FACIT-Sp to examine the relationship of spiritual well-being with depression, hopelessness, and hastened death. In a study by McClain et al. [34], 160 patients with a life expectancy of less than three months were interviewed in order to assess the role of spiritual well-being and the management of suicidal symptomology as a function of spiritual well-being Spearman correlations indicated that the FACIT-Sp was significantly correlated with outcome variables related to depression $(r=-0.68 ; p<0.0001)$. Regression analyses found that the meaning subscale of the FACIT-Sp was significantly related to end-of-life despair (Beta $=-0.44$ for hopelessness, $\mathrm{p}<0.0001 ;-0.44$ for desired for hastened death, $\mathrm{p}<0.001$, and -0.84 for suicidal ideation, $p=0.019$ ), and that it was able to predict suicidal ideation independent of the other measures. The researchers concluded that this subscale also had strong associations with hopelessness and that the FACIT-Sp in general "taps a unique aspect of psychological functioning and is not simply a proxy for depression" (p. 1607).

While a number of studies exist linking spirituality, religiosity and psychological well-being, little research has been done specifically linking spiritual well-being and belief in afterlife. A study was conducted using the FACIT-Sp with a sample of 276 palliative care patients in order to assess if any differences existed between groups of patients who reported being believers in an afterlife and those who did not [35]. Participants were administered a variety of self-report and clinician-rated measurements. Belief in afterlife was measured with three questions: "Do you believe in an afterlife?" "Are your beliefs about an afterlife comforting to you?" and "Are your beliefs about an afterlife distressing to you?" There were no significant differences found between anxiety and depression across the different afterlife groups, but hopelessness was significant at the 0.01 level and desire for hastened death and suicidal ideation were significant at the 0.05 level. This pattern also held true when controlling for age. Further analyses showed that when spiritual well-being levels were controlled for, the associations between afterlife beliefs and the indices of psychological distress were no longer significant. The authors concluded that spiritual well-being is a more robust predictor of psychological functioning than belief in an afterlife. 
In a study of terminally ill cancer patients, Daugherty et al. assessed spiritual well-being using the FACIT-Sp with patients who volunteered for experimental clinical trials [36]. The authors investigated four questions: Are patients who volunteer for phase I clinical trials more or less spiritual than other patients with advanced cancer? What is the role of spirituality in phase I patients' quality of life? Is spirituality associated with phase I patients' realism about their prognosis? Does an association exist between patients' spirituality and medical decision-making preferences? Advanced cancer patients who volunteered for phase I trials $(\mathrm{N}=162)$ were administered the FACIT-Sp as well as other measures. In a multivariable model, phase I patients had slightly higher levels of spiritual well-being $(\mathrm{p}<0.001)$ than a group of 156 advanced cancer patients who were not participants in phase I trials. Spiritual well-being was also found to be positively associated with HRQOL for the phase I patients (Spearman rho $=0.36, \mathrm{p}<0.001$ ). No association, however, was found between spiritual well-being and phase I patients' awareness of their prognosis or decision making preferences.

Health care professionals at all levels are often faced with having to understand the implications of their patients' spiritual beliefs, and for nurses who may be required to provide spiritual care to patients close to death, little is known of the impact spiritual well-being has on the ability to cope when being near end-of-life. One study examining the relationship between spiritual well-being and coping at endof-life explored the role of spiritual well-being in decision-making for patients in a hospice setting [37]. Sixty elder adults (mean age of 63) enrolled in hospice care completed the FACIT-Sp as well as the Serenity Scale and the Cognitive Coping Scale. Spiritual well-being was found to have a significant correlation (at the $\mathrm{p}<0.01$ level) with importance of religion $(r=0.573)$, serenity $(r=0.556)$, peacefulness $(r=0.540)$, comfort $(r=0.399)$, and cognitive coping $(r=0.333)$. Linear regression analyses showed that spiritual well-being was a predictor of peacefulness (accounting for almost $28 \%$ of the variance) for patients nearing the end of their lives. Seventy-three percent of participants reported that illness had strengthened their spiritual well-being and increased religious practices as well, but many also reported low levels of comfort and peace. The authors concluded that if spiritual well-being is helpful in creating a peaceful death among patients, healthcare providers must find a way to address the spiritual needs of the dying and promote a more peaceful death experience.

\section{Conclusions}

Since its development a little over a decade ago, the FACIT-Sp has been used in numerous published studies of individuals diagnosed with and treated for serious illnesses. Although our review has highlighted the significant findings of the work to date, several limitations emerged from the studies reviewed. Surprisingly, all but one of the studies reviewed used cross-sectional designs, which served to limit any conclusions regarding causal factors or observations about changes in spiritual well-being over time. Moreover, this also inhibited the ability to examine the role of adjustment across the illness trajectory (diagnosis, treatment, post-treatment, etc.). Many of these studies conceptualized spiritual well-being as an independent or predictor variable, but under certain conditions, spiritual well-being may function as a process variable or even as a dependent or outcome variable. Greater sophistication in study designs can help disentangle this.

While some of the studies included large sample sizes, many of the samples consisted of individuals with significant heterogeneity with respect to disease type and stage of illness. For example, many of 
the studies of cancer patients included patients with different types of cancer at various time points in their treatment and/or recovery. Confounding these important disease characteristics may obscure unique relationships between spiritual well-being and health outcomes for different types of illnesses or stages of disease. Common findings that emerged across multiple studies were the significant associations among spiritual well-being and indices of psychological well-being. While positive associations between the meaning and peace subscale and emotional well-being are perhaps not surprising, spiritual well-being cannot be "explained away" by reducing it to purely affective states [6]. Spiritual well-being accounts for incremental variance in HRQOL above and beyond the variance accounted for by mood states [13]. So while there is certainly shared variance between spiritual wellbeing and mood states, there is also unique variance in spiritual well-being unexplained by mood states [15]. Studies that include examples of discriminant validity measures may improve our understanding of the unique aspects of spiritual well-being.

Additional limitations were that only four studies included racially or ethnically diverse samples [13,15,18,27] and only one study included a healthy comparison group [19]. Research has demonstrated racial and ethnic differences on various religious and spiritual factors [38-40] and these differences may be associated with important differences in health outcomes. Similarly, lack of adequate control groups prevents us from drawing appropriate conclusions representing adjustment processes unique to patients with serious illnesses. Finally, few of the studies articulated a well-defined theory that served to guide the research. Research conducted without a theoretical framework may be susceptible to methodological oversights and can often amount to "fishing expeditions" and numerous post-hoc explanations. Extending and or modifying existing theoretical models of psychological adjustment to illness, such as the social-cognitive processing theory $[41,42]$ or any of the various health-belief models [43], to include spiritual well-being would represent an advance in this research area.

This review suggests several directions for future research that can contribute to the ongoing knowledge base of spiritual well-being as measured by the FACIT-Sp. More methodologically sound studies are needed. By incorporating longitudinal designs that are grounded in theory and use a wider variety of clinical and behavioral outcomes (e.g., symptom reports, health behaviors), our understanding of these complex relationships will increase. Longitudinal designs would also enable investigation of adjustment across the illness trajectory. Given fluctuating levels of distress and different physical and psychosocial challenges emerging over time, ongoing attention is needed in this area. In the absence of longitudinal designs, more sophisticated analytic strategies such as structural equation modeling would provide an opportunity to identify plausible causal relationships among numerous physical and mental health variables. Moving beyond simple bivariate relationships to examining the role of third variables in the link between spiritual well-being and health is also important. Efforts have been made to identify and explore the mechanisms through which spiritual well-being operates, but ongoing work needs to be done examining the role of spiritual well-being as a possible mediator or moderator of these relationships. Finally, spiritual well-being can be meaningfully understood as an outcome as well as a process variable and more research is necessary to identify the factors that lead to changes in spiritual well-being.

While work in the field of health-related spiritual well-being progresses, new insights surface with each new study, and assessing the role spiritual well-being plays with regards to health status appears 
to be an important consideration related to patient-centered care and better psychosocial outcomes. In the studies summarized above, the FACIT-Sp has shown that spiritual well-being con be considered a core domain in HRQOL assessment, and growing evidence supports its clinical utility in helping to uncover linkages between spiritual well-being, health status, and health outcomes. As a result, our understanding of the role that spirituality plays with regards to living with a chronic illness has the potential to impact cross-cultural understanding of pain and symptom management. Continued research in this area is needed and will allow the field to more fully identify, understand, and appreciate the clinical significance of spiritual well-being for patients with acute and chronic illnesses.

\section{Acknowledgements}

The authors would like to thank all of our colleagues in the Department of Medical Social Sciences at Northwestern University's Feinberg School of Medicine, as well as Lauren Lent and all of the members of the FACIT team.

\section{References}

1. Hill, P.C.; Pargament, K.I.; Hood, R.W.; McCullough, M.E.; Swyers, J.P.; Larson, D.B.; Zinnbauer, B.J. Conceptualizing religion and spirituality: Points of commonality, points of departure. J. Theory Soc. Behav. 2000, 30, 51-78.

2. Heelas, P.; Woodhead, L. The Spiritual Revolution Why Religion is Giving Way to Spirituality; Blackwell Publishing: Malden, MA, USA, 2005.

3. Balboni, T.A.; Vanderwerker, L.C.; Block, S.D.; Paulk, M.E.; Lathan, C.S.; Peteet, J.R.; Prigerson, H.G. Religiousness and Spiritual Support Among Advanced Cancer Patients and Associations With End-of-Life Treatment Preferences and Quality of Life. J. Clin. Oncol. 2007, $25,555-560$.

4. Zinnbauer, B.J.; Pargament, K.I.; Scott, A.B. The emerging meanings of religiousness and spirituality: Problems and prospects. J. Pers. 1999, 67, 889-919.

5. Fetzer Institute; National Institute on Aging. Multidimensional Measurement of Religiousness/Spirituality for Use in Health Research: A Report of the Fetzer Institute/National Institute on Aging Working Group; Fetzer Institute: Kalamazoo, MI, USA, 1999.

6. Koenig, H.G.; McCullough, M.E.; Larson, D.B. Handbook of Religion and Health; Oxford University Press: Oxford, UK, 2001.

7. Fitchett, G.; Canada, A.L. The role of religion/spirituality in coping with cancer: Evidence, assessment, and intervention. In Psychooncology, 2nd ed.; Holland, J.C., Breitbart, W.S., Jacobsen, P.B., Eds.; Oxford University Press: New York, NY, USA, 2010; pp. 440-446.

8. Cotton, S.P.; Levine, E.G.; Fitzpatrick, C.M.; Dold, K.H.; Targ, E. Exploring the relationships among spiritual well-being, quality of life, and psychological adjustment in women with breast cancer. Psychooncology 1999, 8, 429-438.

9. Lennon, C.; Burdick, H. The Lexile Framework As An Approach For Reading Measurement And Success. $\quad$ http://www.lexile.com/m/uploads/whitepapers/Lexile-Reading-Measurement-andSuccess-0504_MetaMetricsWhitepaper.pdf (accessed on 25 January 2011). 
10. Peterman, A.H.; Fitchett, G.; Brady, M.J.; Hernandez, L.; Cella, D. Measuring spiritual wellbeing in people with cancer: The Functional Assessment of Chronic Illness Therapy--Spiritual Well-being Scale (FACIT-Sp). Ann. Behav. Med. 2002, 24, 49-58.

11. Cella, D.F.; Tulsky, D.S.; Gray, G.; Sarafian, B.; Linn, E.; Bonomi, A.; Silberman, M.; Yellen, S.B.; Winicour, P.; Brannon, J.; et al. The Functional Assessment of Cancer Therapy scale: Development and validation of the general measure. J. Clin. Oncol. 1993, 11, 570-579.

12. Cotton, S.; Puchalski, C.M.; Sherman, S.N.; Mrus, J.M.; Peterman, A.H.; Feinberg, J.; Pargament, K.I.; Justice, A.C.; Leonard, A.C.; Tsevat, J. Spirituality and religion in patients with HIV/AIDS. J. Gen. Intern. Med. 2006, 21, S5-S13.

13. Brady, M.J.; Peterman, A.H.; Fitchett, G.; Mo, M.; Cella, D. A case for including spirituality in quality of life measurement in oncology. Psychooncology 1999, 8, 417-428.

14. Canada, A.L.; Murphy, P.E.; Fitchett, G.; Peterman, A.H.; Schover, L.R. A 3-factor model for the FACIT-Sp. Psychooncology 2008, 17, 908-916.

15. Murphy, P.E.; Canada, A.L.; Fitchett, G.; Stein, K.; Portier, K.; Crammer, C.; Peterman, A.H. An examination of the 3-factor model and structural invariance across racial/ethnic groups for the FACIT-Sp: A report from the American Cancer Society's Study of Cancer Survivors-II (SCS-II). Psychooncology 2010, 19, 264-272.

16. Peterman, A.; Reeve, C.L.; Winford, E.; Cotton, S.; Salsman, J.; McQuellon, R.; Cella, D.; Tsevat, J. Measuring meaning and peace with the FACIT-Spiritual Well-being scale: Distinction without a difference? 2011, in preparation.

17. Salsman, J.M.; Yost, K.J.; West, D.W.; Cella, D. Spiritual well-being and health-related quality of life in colorectal cancer: A multi-site examination of the role of personal meaning. Support. Care Cancer 2010, doi: 10.1007/s00520-010-0871-4.

18. Dapueto, J.J.; Servente, L.; Francolino, C.; Hahn, E.A. Determinants of quality of life in patients with cancer. Cancer 2005, 103, 1072-1081.

19. Andrykowski, M.A.; Bishop, M.; Hahn, E.A.; Cella, D.; Beaumont, J.; Brady, M.; Horowitz, M.; Sobocinski, K.A.; Rizzo, J.D.; Wingard, J.R. Long-term health-related quality of life, growth, and spiritual well-being after hematopoietic stem-cell transplantation. J. Clin. Oncol. 2005, 23, 599-608.

20. Krupski, T.L.; Kwan, L.; Fink, A.; Sonn, G.A.; Maliski, S.; Litwin, M.S. Spirituality influences health related quality of life in men with prostate cancer. Psychooncology 2006, 15, 121-131.

21. Nelson, C.J.; Rosenfeld, B.; Breitbart, W.; Galietta, M. Spirituality, Religion, and Depression in the Terminally Ill. Psychosomatics 2002, 43, 213-220.

22. Szaflarski, M.; Ritchey, P.; Leonard, A.; Mrus, J.; Peterman, A.; Ellison, C.; McCullough, M.; Tsevat, J. Modeling the effects of spirituality/religion on patients' perceptions of living with HIV/AIDS. J. Gen. Intern. Med. 2006, 21, S28-S38.

23. Martensson, G.; Carlsson, M.; Lampic, C. Do nurses and cancer patients agree on cancer patients' coping resources, emotional distress and quality of life? Eur. J. Cancer Care 2008, 17, 350-360.

24. Lampic, C.; von Essen, L.; Peterson, V.W.; Larsson, G.; Sjoden, P.O. Anxiety and depression in hospitalized patients with cancer: Agreement in patient-staff dyads. Cancer Nurs. 1996, 19, 419-428. 
25. Lundmark, M. Attitudes to spiritual care among nursing staff in a Swedish oncology clinic. J. Clin. Nurs. 2006, 15, 863-874.

26. Whitford, H.S.; Olver, I.N.; Peterson, M.J. Spirituality as a core domain in the assessment of quality of life in oncology. Psychooncology 2008, 17, 1121-1128.

27. Zavala, M.W.; Maliski, S.L.; Kwan, L.; Fink, A.; Litwin, M.S. Spirituality and quality of life in low-income men with metastatic prostate cancer. Psychooncology 2009, 18, 753-761.

28. Tate, D.G.; Forchheimer, M. Quality of life, life satisfaction, and spirituality: Comparing outcomes between rehabilitation and cancer patients. Am. J. Phys. Med. Rehabil. 2002, 81, 400-410.

29. Edmondson, D.P.; Park, C.L.; Blank, T.O.; Fenster, J.R.; Mills, M.A. Deconstructing spiritual well-being: Existential well-being and HRQOL in cancer survivors. Psychooncology 2008, 17, 161-169.

30. Trevino, K.M.; Pargament, K.I.; Leonard, A.C.; Caprini-Faigin, C.A.; Tsevat, J. Religious Coping and Physiological, Psychological, Social, and Spiritual Outcomes in Patients with HIV/AIDS: Cross-sectional and Longitudinal Findings. AIDS Behav. 2010, 14, 379-389.

31. Perkins, E.A.; Small, B.J.; Balducci, L.; Extermann, M.; Robb, C.; Haley, W.E. Individual differences in well-being in older breast cancer survivors. Crit. Rev. Oncol. Hematol. 2007, 62, 74-83.

32. Yanez, B.; Edmonson, D.; Stanton, A.L.; Park, C.L.; Kwan, L.; Ganz, P.A.; Blank, T.O. Facets of spirituality as predictors of adjustment to cancer: Relative contributions of having faith and finding meaning. J. Consult Clin. Psychol. 2009, 77, 730-741.

33. Rodin, G.; Zimmermann, C.; Rydall, A.; Jones, J.; Shepherd, F.A.; Moore, M.; Fruh, M.; Donner, A.; Gagliese, L. The Desire for Hastened Death in Patients with Metastatic Cancer. J. Pain Symptom Manage. 2007, 33, 661-675.

34. McClain, C.S.; Rosenfeld, B.; Breitbart, W. Effect of spiritual well-being on end-of-life despair in terminally-ill cancer patients. Lancet 2003, 361, 1603-1607.

35. McClain-Jacobson, C.; Rosenfeld, B.; Kosinski, A.; Pessin, H.; Cimino, J.E.; Breitbart, W. Belief in an afterlife, spiritual well-being and end-of-life despair in patients with advanced cancer. Gen. Hosp. Psychiat. 2004, 26, 484-486.

36. Daugherty, C.K.; Fitchett, G.; Murphy, P.E.; Peterman, A.H.; Banik, D.M.; Hlubocky, F.; Tartaro, J. Trusting God and medicine: Spirituality in advanced cancer patients volunteering for clinical trials of experimental agents. Psychooncology 2005, 14, 135-146.

37. Kruse, B.G.; Ruder, S.; Martin, L. Spirituality and Coping at the End of Life. J. Hosp. Palliat. Nurs. 2007, 9, 296-304.

38. Moadel, A.; Morgan, C.; Fatone, A.; Grennan, J.; Carter, J.; Laruffa, G.; Skummy, A.; Dutcher, J. Seeking meaning and hope: Self-reported spiritual and existential needs among an ethnicallydiverse cancer patient population. Psychooncology 1999, 8, 378-385.

39. Mickley, J.; Soeken, K. Religiousness and hope in Hispanic- and Anglo-American women with breast cancer. Oncol. Nurs. Forum 1993, 20, 1171-1177.

40. Nelson, P.B. Ethnic differences in intrinsic/extrinsic religious orientation and depression in the elderly. Arch. Psychiatr. Nurs. 1989, 3, 199-204 
41. Lepore, S.J. A social-cognitive processing model of emotional adjustment to cancer. In Psychosocial Interventions for Cancer; Baum, A., Andersen, B.L., Eds.; American Psychological Association: Washington, DC, USA, 2001; pp. 99-116.

42. Lepore, S.J.; Helgeson, V.S. Social constraints, instrusive thoughts, and mental health after prostate cancer. J. Soc. Clin. Psychol. 1998, 17, 89-106.

43 Weinstein, N.D. Testing four competing theories of health-protective behavior. Health Psychol. 1993, 12, 324-333.

(C) 2011 by the authors; licensee MDPI, Basel, Switzerland. This article is an open access article distributed under the terms and conditions of the Creative Commons Attribution license (http://creativecommons.org/licenses/by/3.0/). 